\title{
Study of the Standard JPEG2000 in Image Compression
}

\author{
S.Medouakh \\ LESIA Laboratory of Research, \\ Electronic Department, \\ University Mohamed Khider of Biskra, Algeria
}

\author{
Z-E. Baarir \\ LESIA Laboratory of Research, \\ Electronic Department, \\ University Mohamed Khider of Biskra, Algeria
}

\begin{abstract}
JPEG2000 is the new standard for the compression of images, which succeeds to JPEG. This standard is motivated primarily by the need for compressed image representations that offer new features increasingly demanded by modern applications, and also offering superior compression performance, especially at low bit-rates. This new standard has many features and characteristics (region of interest, several types of decompression). But these characteristics are accompanied by a much higher algorithmic complexity than JPEG (about five times more complex). In this paper we are interested on studying the algorithm of JPEG2000 and the most complex in the JPEG2000 compression process, the EBCOT entropy encoder, and its performance are presented.
\end{abstract}

\section{Keywords}

Image compression, JPEG2000, JPEG, Wavelet transforms, EBCOT.

\section{INTRODUCTION}

JPEG2000 is the new international standard for image compression [1][11] developed jointly by the International Organization for Standardization (ISO) , and the International Electrotechnical Commission (IEC) and also recommended by International Telecommunications Union (ITU). With the continual expansion of multimedia and Internet applications, the needs and requirements of the technologies used grew and evolved. In March 1997, a new call for contributions was launched for the development of a new standard for the compression of still images, the JPEG 2000 standard [7].

The JPEG 2000 standard provides a set of features that are of importance to many high-end and emerging applications by taking advantage of new technologies. Some of the most important features that this standard should possess are the following [1][5][11] :

* Improved compression efficiency.

* lossy to lossless compression.

* Multiple resolution representation.

* Embedded bit-stream ( progressive decoding and SNR scalability ).

* Tiling.

* Region-of-interest (ROI) coding.

* Error resilience.

* Random codestream access and processing.

* Improved performance to multiple Compression/ Decompression cycles.

* A more flexible file format.
JPEG2000 has a much higher algorithmic complexity. The complexity of the entropy encoder is the most significant in JPEG2000. Which alone constitutes about $70 \%$ of the overall processing time for compression of an image. The principal advantage of entropy coding: EBCOT is creating a highly scalable compressed bitstream, which can be accessed randomly. As other image compression algorithms such as SPIHT [9] and EZW [10], EBCOT uses the wavelet transform to divide the energy of the original image into subbands.

This paper is organized as follows. In section 2, we explain the encoder engine for the JPEG2000. In Section 3 shows experimental results The Obtained using JPEG2000, a comparison with JPEG and SPIHT coder, and establishes the results for different characteristics of JPEG2000. Finally, section 4 contains the conclusion.

\section{JPEG2000 CODEC STRUCTURE}

The general codec structure of JPEG2000 is illustrated in Figure.1, with the form of the encoder given by Figure.1(a) and the decoder given by Figure.1(b). The decoder structure is basically a reverse process of the encoder, with the exception of rate control.

\subsection{Preprocessing}

The first step in preprocessing is tiling. In this step, the source image is partitioned into rectangular non-overlapping tiles if the image is very large [1]. All tiles have exactly the same dimensions, except may be those at the boundary of the image [9]. Each tile is compressed independently. Tiling is an optional process, where it is used for reduction of memory requirements and efficient extraction of a region of the image. The codec expects [2] its input sample data to have a nominal dynamic range that is approximately centered about zero. Suppose that a particular component has $\mathrm{P}$ bits/sample. The samples may be either signed or unsigned, leading to a nominal dynamic range of $\left[-2^{\mathrm{P}-1 ;} 2^{\mathrm{P}-1}-1\right]$ or $\left[0 ; 2^{\mathrm{P}}-1\right]$, respectively. If the sample values are unsigned, the nominal dynamic range is adjusted by subtracting a bias of $2^{\mathrm{P}-1}$ from each of the sample values.

If the sample values are signed, the nominal dynamic range is already centered about zero. By ensuring that the nominal dynamic range is centered about zero, a number of simplifying assumptions can be made in the design of the codec (e.g., with respect to context modeling, numerical overflow, etc...).

\subsection{Intercomponent Transform}

In the encoder, the preprocessing step is followed by the component Transformations step. It is effective in reducing the correlations amongst the multiple components in a multicomponent image. This results in reduction in redundancy and increase in compression performance. The JPEG2000 Part 1 


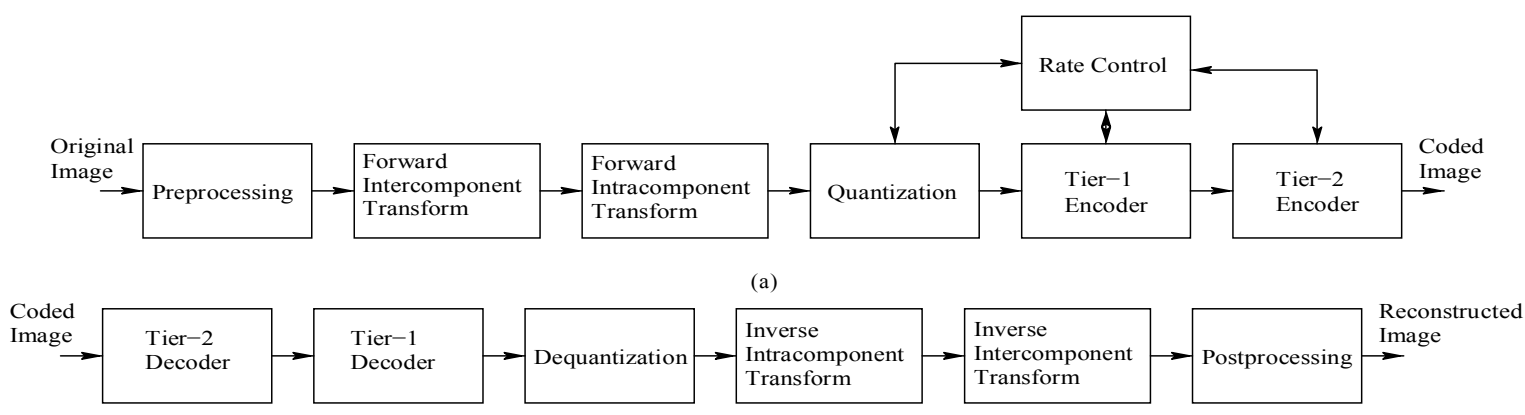

(b)

Fig. 1. Codec structure. The structure of the (a) encoder and (b) decoder.

standard supports two different transformations: (1) reversible color transform (RCT), and (2) irreversible color transform (ICT) [1][2][8][11]. Both of these transforms essentially map image data from the $\mathrm{RGB}$ to $\mathrm{YCrCb}$ color space.

The irreversible color transformation (ICT) is used for lossy compression with the irreversible wavelet transform. The forward and the inverse ICT transformations are represented by the equations 1 (a) and $1(\mathrm{~b})$, respectively.

$$
\begin{gathered}
\left(\begin{array}{l}
Y \\
C_{b} \\
C_{r}
\end{array}\right)=\left(\begin{array}{ccc}
0.299 & 0.587 & 0.114 \\
-0.16875 & -0.33126 & 0.5 \\
0.5 & -0.41869 & -0.08131
\end{array}\right) \cdot\left(\begin{array}{l}
R \\
G \\
B
\end{array}\right) \\
\left(\begin{array}{l}
R \\
G \\
B
\end{array}\right)=\left(\begin{array}{ccc}
1.0 & 0 & 1.402 \\
1.0 & -0.34413 & -0.71414 \\
1.0 & 1.772 & 0
\end{array}\right) \cdot\left(\begin{array}{l}
Y \\
C_{b} \\
C_{r}
\end{array}\right)
\end{gathered}
$$

The reversible color transformation (RCT) is used for lossless or lossy compression with the reversible wavelet transform. The forward and inverse RCT transformations are represented by the Equations 2(a) and 2(b) respectively:

$$
\begin{aligned}
\left(\begin{array}{l}
Y_{r} \\
V_{r} \\
U_{r}
\end{array}\right) & =\left(\begin{array}{c}
\left.\frac{R+2 G+B}{4}\right\rfloor \\
R-G \\
B-G
\end{array}\right) \\
\left(\begin{array}{l}
G \\
R \\
B
\end{array}\right) & =\left(\begin{array}{c}
Y_{r}-\left\lfloor\frac{U_{r}+V_{r}}{4}\right\rfloor \\
V_{r}+G \\
U_{r}+G
\end{array}\right)
\end{aligned}
$$

\subsection{Discrete Wavelet Transformation}

The block DCT transformation in baseline JPEG has been replaced with the full frame DWT in JPEG2000 [8][14][15].

After component transform, the tile-components are decomposed into different decomposition levels using discrete wavelet transform (DWT). These decomposition levels contain a number of subbands, which consist of coefficients that describe the horizontal and vertical spatial frequency characteristics of the original tile component [11]. The DWT can be irreversible or reversible. The default irreversible transform is implemented by a Daubechies 9-tap/7-tap filter [3]. The default reversible transformation is implemented by a 5-tap/3-tap filter [4][11].

The standard can support two filtering modes: convolution based and lifting based. Convolution-based filtering consists in performing a series of dot products between the two filters and the signal. Lifting-based filtering consists of a sequence of very simple filtering operations for which alternately odd sample values of the signal are updated with a weighted sum of even sample values, and even sample values are updated with a weighted sum of odd sample values (see Figure 2) [4][11][16]. The lifting-based filtering for the $5 / 3$ analysis filter are computed by equation (3):

$$
\begin{aligned}
Y(2 n+1) & =X(2 n+1)-\left\lfloor\frac{X(2 n)+X(2 n+2)}{2}\right\rfloor \\
Y(2 n) & =X(2 n)+\left\lfloor\frac{Y(2 n-1)+Y(2 n+1)+2}{4}\right\rfloor
\end{aligned}
$$

Where $X$ is the input signal and $Y$ is the output signal. And the corresponding inverse transformation for the reversible $5 / 3$ synthesis filter is computed by Equation 4 :

$$
\begin{aligned}
& X(2 n)=Y(2 n)-\left\lfloor\frac{Y(2 n-1)+Y(2 n+1)+2}{4}\right\rfloor \\
& X(2 n+1)=Y(2 n+1)+\left\lfloor\frac{X(2 n)+X(2 n+2)}{2}\right\rfloor
\end{aligned}
$$

Where $Y$ is the input signal, and $X$ is the output signal. For irreversible 9/7 analysis filter, Equation 5 describes the 4 "lifting" steps (STEPS 1 through 4) and the 2 "scaling" steps (STEPS 5 through 6) of the 1D filtering performed on the signal $X$ to produce the coefficients of signal $Y$.

$$
\begin{array}{lc}
Y(2 n+1) \leftarrow X(2 n+1)+(\alpha \times[X(2 n)+X(2 n+2]) & {[\text { step } 1]} \\
Y(2 n) \leftarrow X(2 n)+(\beta \times[Y(2 n-1)+Y(2 n+1]) & {[\text { step } 2]} \\
Y(2 n+1) \leftarrow Y(2 n+1)+(\gamma \times[Y(2 n)+Y(2 n+2]) & {[\text { step } 3]} \\
Y(2 n) \leftarrow Y(2 n)+(\delta \times[Y(2 n-1)+Y(2 n+1]) & {[\text { step } 4]} \\
Y(2 n+1) \leftarrow-K \times Y(2 n+1) & {[\text { step5 }]} \\
Y(2 n) \leftarrow(1 / K) \times Y(2 n) & {[\text { step } 6]}
\end{array}
$$




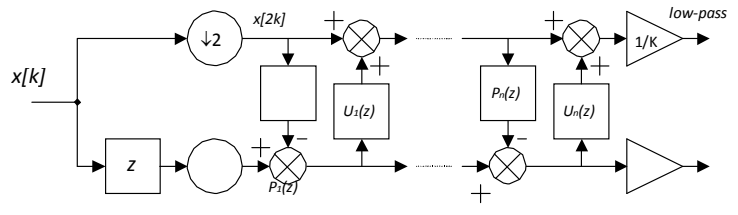

Fig.2: The forward (analysis) wavelet transform using lifting. $\mathrm{P}$ and $\mathrm{U}$ stand for prediction and update, respectively.

Where the values of the parameters are:

$$
\left\{\begin{array}{l}
\alpha=-1.586134342 \\
\beta=-0.052980118 \\
\gamma=0.882911075 \\
\delta=0.443506852
\end{array}\right.
$$

and the scaling factor $K$ is equal to: $K=1.230174105$.

Also, the irreversible inverse transformation of the Daubechies $9 / 7$ synthesis filter is computed by Equation 6 :

$$
\begin{array}{ccc}
X(2 n) \leftarrow K \times Y(2 n) & {[\text { step } 1]} & \\
X(2 n+1) \leftarrow-(1 / K) \times Y(2 n+1) & {[\text { step } 2]} & \\
X(2 n) \leftarrow X(2 n)-(\delta \times[X(2 n-1)+X(2 n+1]) & {[\text { step } 3]} \\
X(2 n+1) \leftarrow X(2 n+1)-(\gamma \times[X(2 n)+X(2 n+2]) & {[\text { step } 4]} \\
X(2 n) \leftarrow X(2 n)+(\beta \times[X(2 n-1)+X(2 n+1]) & {[\text { step }]} \\
X(2 n+1) \leftarrow X(2 n+1)-(\alpha \times[X(2 n)+X(2 n+2]) & {[\text { step } 6]}
\end{array}
$$

Where the values of the parameters and the scaling factor $K$ are the same as used in Equation 3.

The lifting steps corresponding to the $(5 / 3)$ filter-bank and (9/7) filter- bank are shown in Figure 3.
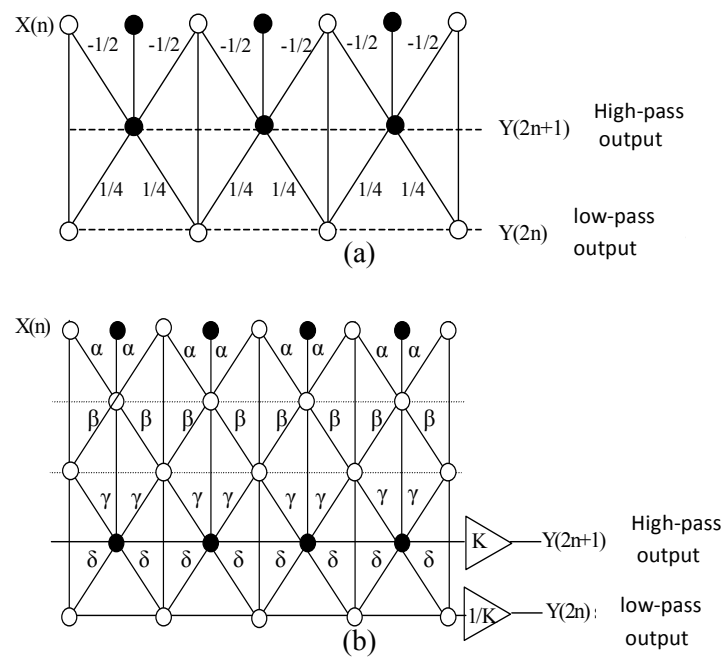

Fig.3. Lifting prediction /update steps:

(a).for the $(5 / 3)$ filter-bank.

(b).for the (9/7) filter-bank.

\subsection{Quantization}

The transform coefficients are quantized with a Uniform scalar quantization with dead-zone. Quantiza-tion is the process by which the coefficients are reduced in precision [5][8][11]. Each of the transform coefficients $c_{b}(u, v)$ of the subband $b$ is quantized to the value $q_{b}(u, v)$ according to the formula :

$$
q_{b}(u, v)=\operatorname{signe}\left(c_{b}(u, v)\right)\left\lfloor\frac{\left|c_{b}(u, v)\right|}{\Delta_{b}}\right\rfloor
$$

For the reversible transform ( $5 / 3$ filter), the quantization stepsize $\Delta_{\mathrm{b}}$ for each subband $\mathrm{b}$ is required to be one, which means no lossy quantization performed. However, for the irreversible transform ( $9 / 7$ filter), the quantization step sizes for all subbands are retrieved from the following equation:

$$
\Delta_{b}=\left(1+\frac{\mu_{b}}{2^{11}}\right) \cdot 2^{R b-\varepsilon b}
$$

It is represented relative to the nominal dynamic range $R_{b}$, the exponent coefficient $\varepsilon_{\mathrm{b}}$, and the mantissa coefficient $\mu_{\mathrm{b}}$ of each subband $b$

\subsection{Entropy Encoding}

The entropy coding and generation of compressed bitstream in JPEG2000 is divided into two coding steps: Tier-1 and Tier-2 coding [1].

- Tier-1 (EBCOT coder [12][13] and Binary Arithmetic Coding-MQ-Coder .

- Tier-2 (Organization of the bit-stream).

\subsubsection{Tier-1 Coding}

After quantization, each subband is divided into rectangular blocks, called code-blocks (see Figure 4); these code-blocks are encoded independently. The code-block is decomposed into $P$ bit-planes and they are encoded from the most significant bit-plane to the least significant bit-plane sequentially (see figure 5). Each bit-plane is first encoded by a fractional bit-plane coding (BPC) mechanism to generate intermediate data in the form of a context and a binary decision value for each bit position. In JPEG2000 the embedded block coding with optimized truncation (EBCOT) algorithm [12] has been adopted for the BPC.

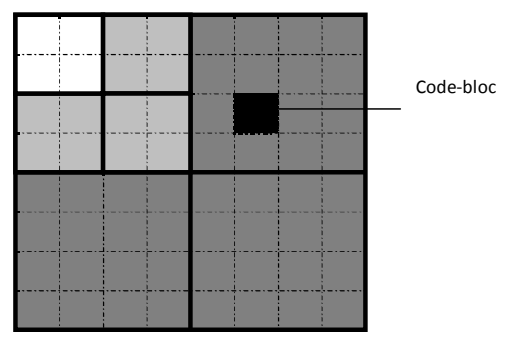

Fig 4 : Partitioning into code-blocks

\subsubsection{Coder EBCOT:}

EBCOT encodes each bit-plane in three coding passes. The three coding passes in the order in which they are performed on each bit-plane are significant propagation pass, magnitude refinement pass, and cleanup pass.

All three types of coding passes scan the samples of a code block in the same fixed order shown in Figure 6 The code block is partitioned into horizontal stripes, each having a nominal height of four samples. As shown in the diagram, the stripes are scanned from top to bottom. Within a stripe, columns are scanned from left to right. Within a column, samples are scanned from top to bottom [5]. 


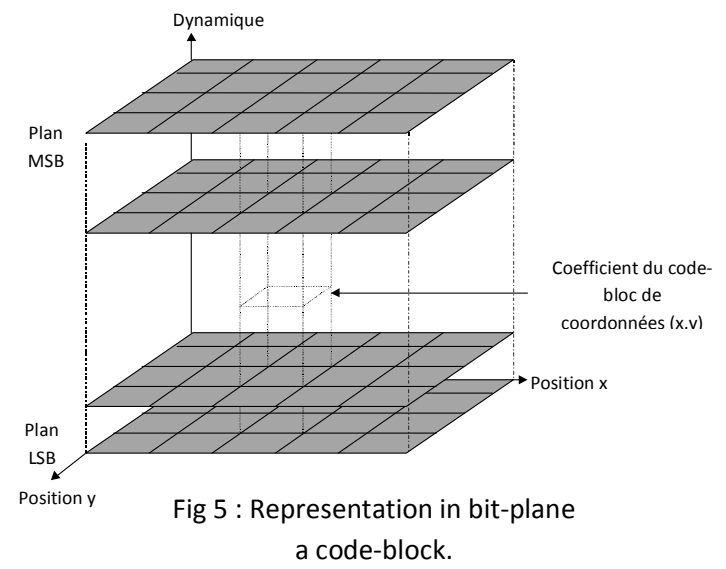

Each coefficient bit in the bit plane is coded in only one of the three coding passes [11], and for each coefficient in a block is assigned a binary state variable called its significance state that is initialized to zero (insignificant) at the start of the encoding. The significance state changes from zero to one (significant) when the first nonzero magnitude bit is found. The context vector for a given coefficient is the binary vector consisting of the significance states of its eight immediate neighbor coefficients [8] For each pass, contexts are created which are provided to the arithmetic coder [11][12][13].

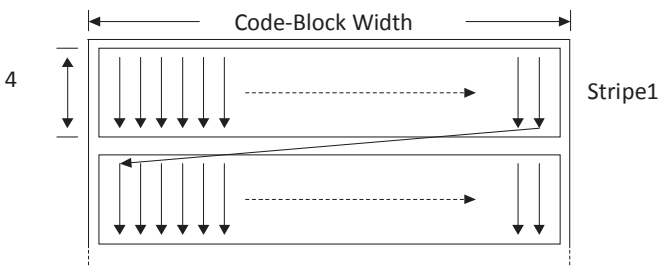

Fig.6. Sample scan order within a code block.

In the following each coding pass is described:

a) Significance propagation pass :

During the significance propagation pass, a bit is coded if its location is not significant, but at least one of its eight-connect neighbors is significant.

b) Magnitude refinement pass :

During this pass, all bits that became significant in a previous biplane are coded. The magnitude refinement pass includes the bits from coefficients that are already significant.

c) Clean-up pass :

The clean-up pass is the final pass in which all bits not encoded during the previous passes are encoded (i.e., coefficients that are insignificant and had the context value of zero during the significance propagation pass). The very first pass in a new code block is always a clean-up pass.

\subsubsection{Binary Arithmetic Coding-MQ-Coder}

As explained in the previous section [1], the fractional bitplane coding (EBCOT) produces a sequence of symbols, pairs of context and decision (CX, D), in each coding pass. The context-based adaptive binary arithmetic MQ-coder that is used in JBIG2 is adapted in JPEG2000 standard to encode these symbols.

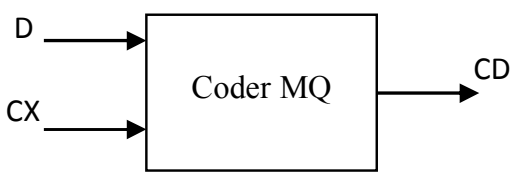

Fig.7 : Inputs and outputs of the encoder arithmetic [7].

\subsubsection{Tier-2 Coding}

The final operation in a JPEG 2000 compression consists in generating the codestream. This one is a collection of codeblock's bit streams gathered into structures called packets and delimited by a header, containing all the signaling needed to decode. The various natural progression modes of JPEG 2000 codestreams are obtained by ordering packets according to a specific progression. Five major progression modes are actually admissible, depending upon which information is interesting to be reconstructed first [8] :

1). Layer-resolution-component-position-progression (LRCP)

2). Resolution-layer-component-position-progression (RLCP)

3). Resolution-position-component-layer-progression (RPCL)

4). Position-component-resolution-layer-progression (PCRL)

5). Component-position-resolution-layer-progression (CPRL)

\subsection{Rate control}

Rate control is responsible for achieving layer bit-rate targets [5]. This can be achieved through two distinct mechanisms: 1) the choice of quantized step sizes, and 2) the selection of the subset of coding passes to include in the code stream.

When the first mechanism is employed, if the step sizes are in creased, the rate decreases, at the cost of greater distortion. Although this rate control mechanism is conceptually simple. The drawback of such a mechanism is that every time the quantizer step size is changed, entire tire-1 encoding must be performed again. Since tier-1 coding requires a considerable amount of computation, this approach to rate control may not be practical in computationally-constrained encoders.

When the second mechanism is used, the encoder can elect to discard coding passes in order to control the rate. The encoder knows the contribution that each coding pass makes to rate, and can also calculate the distortion reduction associated with each coding pass. Using this information, the encoder can then include the coding passes in order of decreasing distortion reduction, until the desired rate is achieved.

\section{EXPERIMENTAL RESULTS}

In this section, we briefly demonstrate the compression performance of JPEG2000. We compare JPEG2000 with the traditional JPEG standard and coder SPIHT. To obtain the reported results the test images, Lena and Goldhill of size (512x512) this images are grayscale. For lossy compression, distortion was characterized by the peak signal to noise ratio ( PSNR ), which for an 8-bit decompressed image is defined as [8]:

$$
\operatorname{PSNR}(d B)=10 \log _{10}\left(\frac{(255)^{2}}{M S E}\right)
$$

Where MSE refers to the mean squared error between the original image and the reconstructed image. 
Table (1) and figure (8) present the results PSNR for each test image and each compression system (JPEG2000, JPEG, SPIHT), JPEG2000 and SPIHT encoder for 5 level decomposition with 9/7 filter, JPEG and SPIHT used the arithmetic coding. We can see that, PSNR values depend very much on image content.

In Figure 8, we can see that the JPEG2000 and SPIHT significantly outperform the JPEG for all test images and all bit-rates (RC), particularly at low bit-rates. The superiority of the JPEG2000 and SPIHT over the existing JPEG can be subjectively judged with the help of Figure 9, where the reconstructed grayscale image "Lena" is shown after compression for low and high bit-rates (RC). It becomes evident that JPEG2000 outperforms JPEG baseline. This performance superiority of the JPEG2000 decreases as the bit rate increases.

From the point of view of visual quality, JPEG2000 and SPIHT is $10 \%$ to $25 \%$ better than the baseline JPEG (for images compressed at approximately $0.5-1 \mathrm{bpp}$ ) [9].

Form these results; we can see that high and moderate bitrates (above $0.75 \mathrm{bpp}$ ) for all test images JPEG produce better visual picture quality than wavelet-based techniques JPEG2000 and SPIHT. At low bit-rates (below 0.5 bpp) JPEG picture quality degrades below JPEG2000 and SPIHT picture quality, because of the artifacts introduced by block-based DCT scheme. In general, we can say that for high quality imaging applications (i.e., $0.5-1.0 \mathrm{bpp}$ ) JPEG2000 is 10 $20 \%$ better than JPEG.

\begin{tabular}{|c|c|c|c|c|}
\hline \multirow{4}{*}{ Image } & \multicolumn{2}{|c|}{ Lena } & \multicolumn{2}{c|}{ Goldhill } \\
\cline { 2 - 5 } Codeur & $\begin{array}{c}\text { RC } \\
\text { (bpp) }\end{array}$ & $\begin{array}{c}\text { PSNR } \\
\text { (dB) }\end{array}$ & $\begin{array}{c}\text { RC } \\
\text { (bpp) }\end{array}$ & $\begin{array}{c}\text { PSNR } \\
\text { (dB) }\end{array}$ \\
\hline \multirow{4}{*}{\begin{tabular}{c} 
JPEG \\
\cline { 2 - 5 }
\end{tabular}} & 0.0324 & 21.2948 & 0.0090 & 19.8118 \\
\cline { 2 - 5 } & 0.0935 & 25.8765 & 0.0561 & 23.0016 \\
\cline { 2 - 5 } & 0.2236 & 30.7456 & 0.2223 & 27.5055 \\
\cline { 2 - 5 } & 0.4790 & 35.6696 & 0.5631 & 31.9329 \\
\cline { 2 - 5 } & 1.0968 & 40.4446 & 1.1729 & 36.9310 \\
\hline \multirow{4}{*}{ JPEG } & 0.1810 & 20.3435 & 0.1354 & 19.3424 \\
\cline { 2 - 5 } & 0.2340 & 26.0567 & 0.2142 & 23.6091 \\
\cline { 2 - 5 } & 0.3243 & 29.7085 & 0.3703 & 26.8707 \\
\cline { 2 - 5 } & 0.5835 & 34.5152 & 0.8943 & 33.4413 \\
\cline { 2 - 5 } & 1.1099 & 38.7461 & 1.2948 & 36.5300 \\
\hline \multirow{5}{*}{ SPIHT } & 0.0396 & 20.0794 & 0.0363 & 19.9559 \\
\cline { 2 - 5 } & 0.1166 & 25.4088 & 0.1596 & 24.5335 \\
\cline { 2 - 5 } & 0.2741 & 30.2508 & 0.4236 & 29.2589 \\
\cline { 2 - 5 } & 0.5315 & 35.2138 & 0.9169 & 34.3403 \\
\cline { 2 - 5 } & 1.0935 & 40.2956 & 1.2647 & 37.1046 \\
\hline
\end{tabular}

Table.1. Comparison of the PSNR between JPEG2000, JPEG and SPIHT; for "Lena" and "Goldhill".

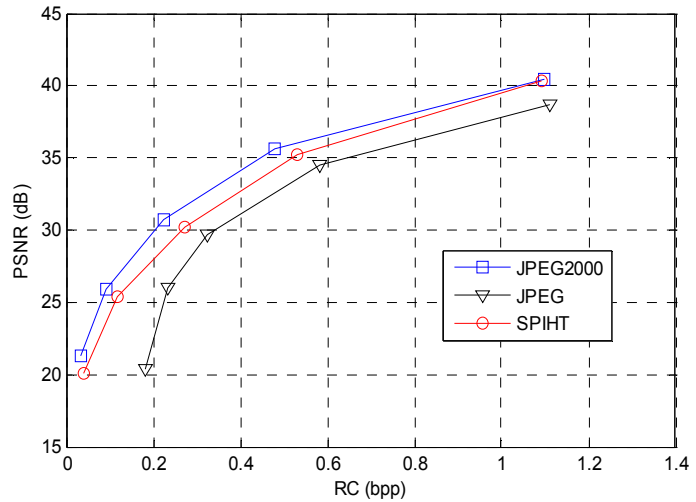

(a)

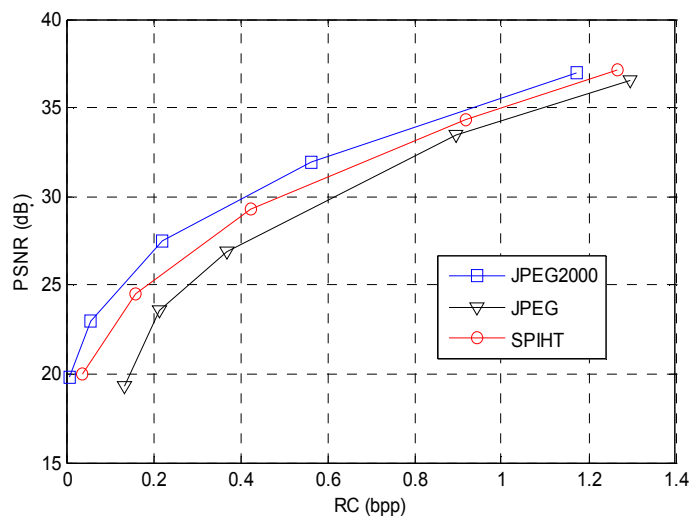

(b)

Fig.8. PSNR results for the lossy compression of different compression standards : (a). Lena, (b). Goldhill

\subsection{Characteristics of JPEG2000}

The new JPEG2000 standard offers new features (ROI, progressivity in quality or resolution) to cope with current needs in the field of imaging, it uses the EBCOT coding highly scalable. In this work, we presented the two characteristics, region of interest (ROI) and progressive in quality or resolution.

\section{Region of interest ROI:}

The functionality of ROI is important in applications where certain parts of the image are more important than others [8][11]. The code of the ROI in Part 1 of the standard is based on a technique called "MAXSHIF [6]. The figure 10 shows two images with and without ROI, respectively. One clearly sees the difference in visual quality in the region of interest for the image "Goldhill".

\section{$\checkmark \quad$ Progressivity in quality or resolution}

JPEG2000 compression system is based on the progressivity. The principal advantage of the compression Progressive is the desired rate or resolution. The two types of progressivity are very important for Internet applications, for example.

Progressivity in quality (SNR), Figure 11 shows the reconstructed images for different layers.

Progressivity in resolution, Figure 12 shows the reconstructed images for different resolutions. 


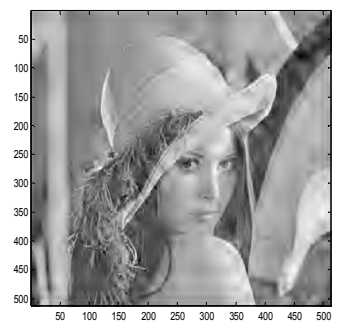

$\mathrm{PSNR}=25.8765 \mathrm{db}, \mathrm{RC}=0.0935 \mathrm{bpp}$ (a)

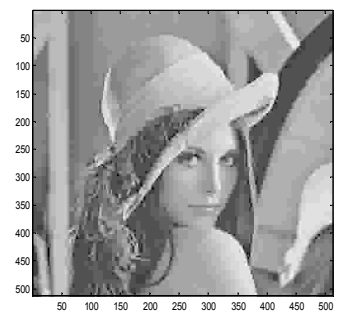

PSNR $=26.0567 \mathrm{db}, \mathrm{RC}=0.2340 \mathrm{bpp}$

(b)

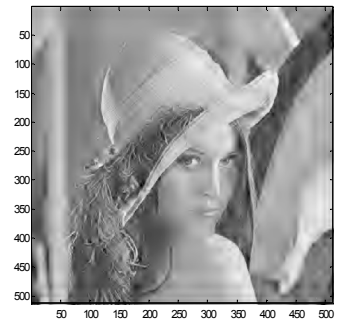

PSNR $=25.4088 \mathrm{db}, \mathrm{RC}=0.1166 \mathrm{bpp}$

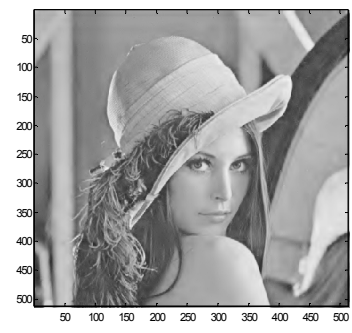

$\mathrm{PSNR}=35.2138 \mathrm{db}, \mathrm{RC}=0.5315 \mathrm{bpp}$

(c)

Fig.9. Images reconstructed " Lena " by means of,

(a). JPEG2000, (b). JPEG, (c) : SPIHT.

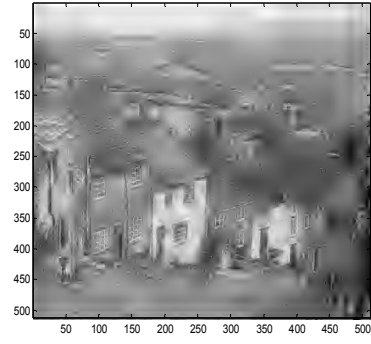

Sans ROI

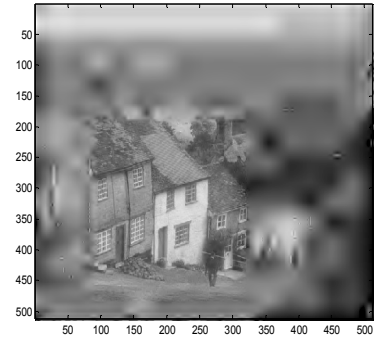

Avec ROI
Fig.10 : Images reconstructed « Goldhill » without ROI et with ROI.

\section{CONCLUSION}

In this paper, we have presented the part1 of the algorithm JPEG2000 standard for image compression. It provides a wide range of functionalities to better address increasing needs for compression. Comparative results have shown that JPEG2000 and SPIHT (DWT) provide superior performance at low bitrates, while blocky artifacts are noticed in JPEG (DCT) images. The algorithm of standard JPEG2000 is more complex than JPEG algorithm. In future work, we propose studying the standard for JPEG2000 using the second generation wavelet transformation (bandelets, curvelets and ridgelets).

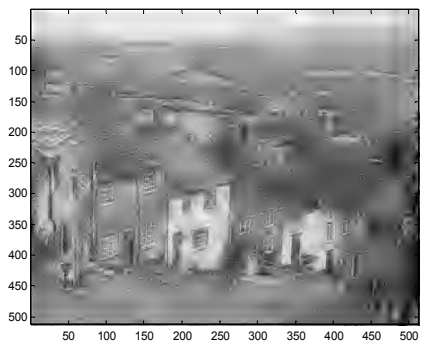

$\mathrm{PSNR}=23.0016 \mathrm{db}, \mathrm{RC}=0.0561 \mathrm{bpp}$

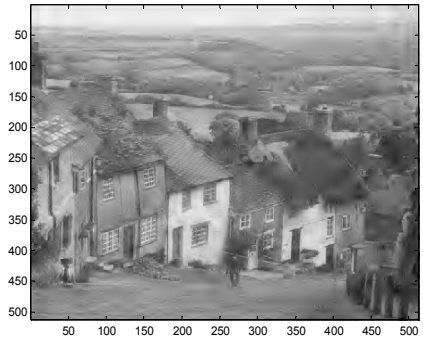

$\mathrm{PSNR}=27.5055 \mathrm{db}, \mathrm{RC}=0.2223 \mathrm{bpp}$

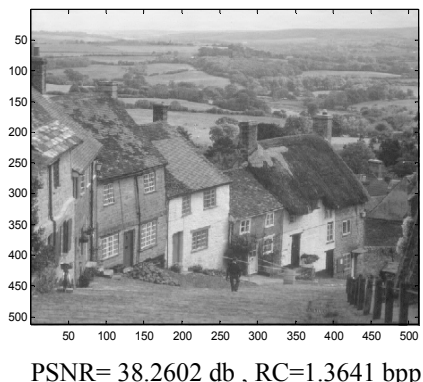

Fig.11 : Images reconstructed « Goldhill » for different layers. 


\section{Résolution 3}
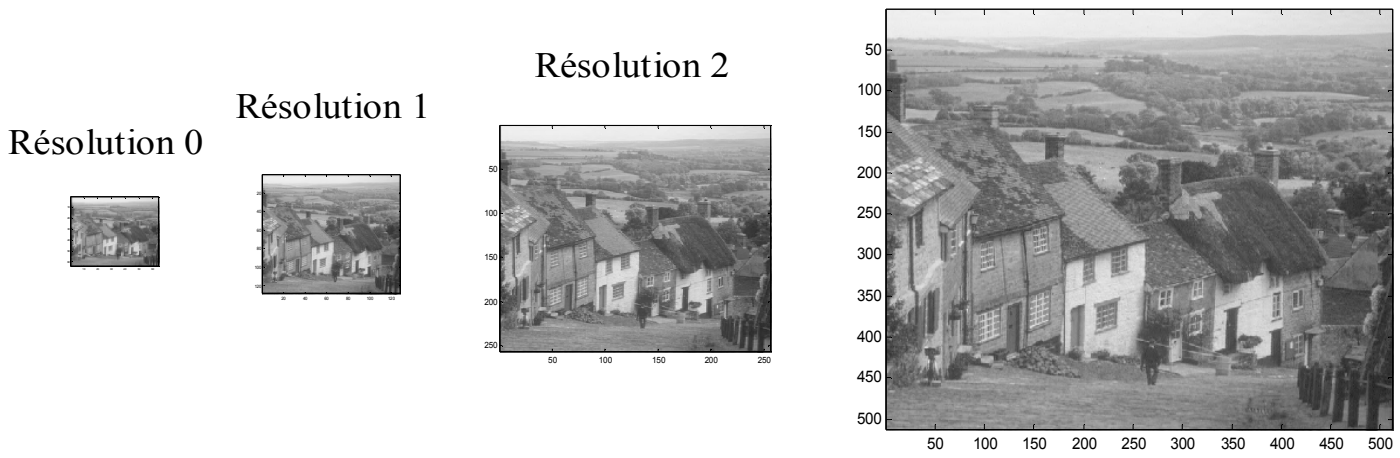

Fig.12: Image reconstructed « Goldhill » for different resolutions.

\section{REFERENCES}

[1] T. Acharya, P.S. Tsai, "JPEG2000 Standard for Image Compression Concepts, Algorithms and VLSI Architectures", John Wiley \& Sons, Hoboken, New Jersey, 2004.

[2] M.D. Adams, "The JPEG2000 Still Image Compression Standard”, ISO/IEC JTC1/SC29/WG1 (ITU-T SG8), 2001.

[3] M. Antonini, M. Barlaud, P. Mathieu, I. Daubechies, "Image Coding Using Wavelet Transform", IEEE Trans. On Image Processing, Vol.1, No.2, pp. 205- 220, Avril 1992.

[4] R.C. Calderbank, I. Daubechies, W. Sweldens, and B.-L. Yeo, "Wavelet Transforms That Map Integers to Integers", Applied and Computational Harmonic Analysis (ACHA), Vol.5, No. 3, pp. 332-369 1998.

[5] C. Christopoulos, A. Skodras, T. Ebrahimi, "The JPEG2000 Still Image Coding System : An Overview", IEEE Transactions on Consumer Electronics, Vol. 46, No. 4, pp.1103-1127, november 2000.

[6] C. Christopoulos, J. Askelöf, M. Larsson, Efficient methods for encoding regions of interest in the upcoming JPEG2000 still image coding standard, Published in IEEE Signal Processing Letters, Vol. 7, No. 9, pp. 247249, September 2000

[7] ISO/IEC 15444-1, "Information Technology JPEG2000 Image Coding System-Part 1: Core Coding System", 2000.

[8] M. Rabbani, R Joshi, "An overview of the JPEG2000 still image compression standard", Signal Processing : Image Communication, Vol. 17, pp. 3-48, 2002.
[9] A. Said, W. A. Peralman, A New Fast and Efficient Image Codec Based on Set Partitioning in Hierarchical Trees, IEEE Trans. on Circuits and Systems for Video Technology, Vol. 6, No. 3, pp. 243-250, June 1996.

[10] J.M. Shapiro, Embedded Image Coding Using Zerotrees of Wavelet Coefficients, IEEE Trans. on Signal Processing, Vol. 41, No. 12, pp. 3445-3462, December 1993.

[11] A. Skodras, C. Christopoulos, T. Ebrahimi, "The JPEG2000 Still Image Compression Standard", IEEE Signal Processing Magazine, pp.36-58, September 2001.

[12] D. Taubman, "High performance scalable image compression with EBCOT", IEEE Trans. Image Processing, vol. 9, pp. 1158-1170, July 2000.

[13] D. Taubman, E. Ordentlich, M. Weinberger, G. Seroussi, I. Ueno, and F. Ono, "Embedded block coding in JPEG $2000 "$, in Proc. IEEE Int. Conf. Image Processing, Vancouver, Canada, Sept. 2000, vol. II, pp. 33-36.

[14] S. Medouakh , Z.E . Baarir, "Compression d'images fixes par la norme JPEG2000" JIG'2007-3 ${ }^{\text {èmés }}$ Journées Internationales sur l'Informatique Graphique , pp 10-15, Constantine, Algérie, oct. 2007.

[15] S. Medouakh, Z.E. Baarir, “JPEG2000 : La nouvelle norme de la compression d'images" 2nd International Conference on Electronics \& Electrical Engineering ICEEE'08, pp 667-671, Laghouat, Algeria, April 2008.

[16] S. Medouakh, Z.E. Baarir, “L'utilisation des filtres 9/7 et $5 / 3$ dans la norme JPEG2000" 6èmés Séminaire Nationales en Informatique Biskra, Mai 2008, pp241245. 